\title{
Similarity Match Over High Speed Time-Series Streams
}

\author{
Xiang Lian ${ }^{\S} \quad$ Lei Chen ${ }^{\S} \quad$ Jeffrey $\mathrm{Xu} \mathrm{Yu}^{\dagger} \quad$ Guoren Wang $^{*} \quad$ Ge $\mathrm{Yu}^{*}$ \\ $\S^{\S}$ Hong Kong University of \\ Science and Technology, \\ Hong Kong, China \\ \{xlian,leichen\}@cse.ust.hk
† The Chinese University of Hong
Kong, Hong Kong, China
yu@se.cuhk.edu.hk
* Northeastern University, China
\{wanggr,yuge\}@mail.neu.edu.cn

\begin{abstract}
Similarity-based time series retrieval has been a subject of long term study due to its wide usage in many applications, such as financial data analysis, weather data forecasting, and multimedia data retrieval. Its original task was to find those time series similar to a pattern (query) time series data, where both the pattern and data time series are static. Recently, with an increasing demand on stream data management, similarity-based stream time series retrieval has raised new research issues due to its unique requirements during the stream processing, such as one-pass search and fast response. In this paper, we address the problem of matching patterns over high-speed stream time series data. We will develop a novel representation, called multi-scaled segment mean (MSM), for stream time series data, which can be incrementally computed and thus perfectly adapted to the stream characteristics. Most importantly, we propose a novel multi-step filtering mechanism over the multi-scaled representation. Analysis indicates that the mechanism can greatly prune the search space and thus offer fast response. Extensive experiments show the multi-scaled representation together with the multi-step filtering scheme can efficiently filter out false candidates and detect patterns, compared to the multiscaled wavelet.
\end{abstract}

\section{Introduction}

Recently, stream time series data management has become a hot research topic due to its wide range of applications, such as Internet traffic analysis [11], sensor network monitoring [30], moving object search and tracking $[10,21]$, which require continuously monitoring stream time series. Compared to traditional archived data, stream time series have their own characteristics: (1) Data are frequently updated in stream time series. Thus, previous methods applied to archived data may not work in this scenario. (2) Due to the frequent updates, it is very difficult to store all the data in memory or on disk, thus, data summarization and one pass algorithms are usually required to achieve a real time response.

In this paper, we deal with an important scenario in stream applications where incoming data are from a set of continuous stream time series and patterns are a fixed set of time series. At each timestamp, a new data item is appended to each stream time series. We want to quickly find all the similar pairs up to the current time, one from stream time series and the other from patterns, so that their distances do not exceed a user-specified threshold $\mathcal{E}$.

One application of this problem is stock data monitoring. Given a set of real-time stock data and a number of pre-defined movement trends (e.g. "two bottom" or "head-shoulder" patterns), the system monitors the stock data and gives a fast response whenever the stock data are similar to the pre-defined patterns. In fact, detecting similar patterns over stream time series can be considered as a typical similarity search problem over datasets defined as follows:

Definition 1 (Similarity Search) Given a time-series dataset $D=\left\{t_{l}, t_{2}, \ldots, t_{n}\right\}$, a query set $Q=\left\{q_{1}, \ldots, q_{m}\right\}$, and a user-specified threshold $\mathcal{E}$, a similarity-search operation retrieves all time series $t_{i}$, so that for some query $q_{j}$, the distance between $t_{i}$ and $q_{j}$ does not exceed $\varepsilon$, where $1 \leq i \leq n, 1 \leq j \leq m$.

Previous works on the similarity-based search over archived time series are not applicable to this problem, since they cannot handle the frequent update efficiently. Current work for searching over stream time series data focus on detecting a single static pattern over multiple stream time series data [6], or checking which pattern (from multiple static patterns) is close to a single stream time series data up to the current timestamp [13]. Wu et al. [29] propose an online matching algorithm for detecting subsequences of financial data over a dynamic database. However, their segmentation and pruning methods are designed for financial data only and thus cannot be applied to detect the general patterns over stream time series. 
In this paper, we propose a novel approach to efficiently perform the search as defined in Definition 1. Specifically, in order to save the computation cost and offer a fast response, we present a novel representation for time series, namely multi-scaled segment mean (MSM). We show that this representation can be incrementally computed, which adapts to the frequent update during stream processing. Furthermore, our MSM representation works very well under all $\mathrm{L}_{p^{-}}$ norms, for $p \geq 1$, which offers users the flexibility of choosing different $p$. Moreover, different norms have their own applications, for example, $L_{1}$-norm is robust against impulse noise [25], $\mathrm{L}_{\infty}$-norm is used for atomic matching [2], and so on. On the other hand, the wellknown multi-scaled representation, wavelets (DWT), can only work under $\mathrm{L}_{2}$-norm (i.e. the Euclidean distance). Most importantly, we propose a multi-step filtering approach to prune the false candidates before computing the real distance between pattern and stream time series. To summarize, the main contributions of this paper are listed as follows:

1. We introduce a novel representation, namely multiscaled segment mean (MSM), for stream time series data, which can be incrementally computed and is thus suitable for stream processing with frequent updates.

2. We propose a multi-step filtering technique, called SS scheme, over the MSM representation of time series data, and show that no false dismissals are introduced after filtering.

3. We give our analytic results indicating when we should use SS scheme and up to which scale we use it.

The rest of the paper is arranged as follows. Section 2 gives a brief review of the related work. Section 3 demonstrates the background and gives the problem definition. Section 4 proposes our multi-scaled segment mean (MSM) representation together with a multi-step filtering algorithm SS. Section 5 evaluates the effectiveness and efficiency of the proposed methods compared to another popular multi-scaled representation, wavelet (DWT). Finally, Section 6 concludes the paper.

\section{Related Work}

In the literature, many research works have been proposed for the similarity-based search over the archived time series data. The pioneering work by Agrawal et al. [1] used the Euclidean distance to measure the similarity between two time series. Specifically, the time series is first transformed to a lower dimensional sequence by Discrete Fourier Transform (DFT) which is a dimensionality reduction technique for time series data, and then inserted into an index structure, that is, R-tree [14]. Faloutsos et al. [12] extended this work to allow the subsequence matching and proposed the GEMINI framework for indexing time series. GEMINI reduces the dimensionality of the time series with any dimensionality reduction technique that follows a lower bounding lemma (i.e. the distance between any reduced series is less than that in the original space), inserts the transformed series into an index, and performs the similarity search through the index. Due to the lower bounding lemma, no false dismissals are introduced.

Subsequent work has focused on two main aspects: new dimensionality reduction techniques and new approaches for measuring the similarity between two time series. Examples of dimensionality reduction techniques include SVD [18], DWT [8], PAA [31], APCA [15], and Chebyshev Polynomials (CP) [7]. However, these techniques have a high percentage of false positives due to their loose lower bounds [9]. Several distance functions have been proposed for different applications, such as Euclidean distance $\left(\mathrm{L}_{2}-\right.$ norm) [1, 12], DTW [4], LCSS [27], and ERP [9]. Euclidean distance requires the time series to have the same length, which restricts its applications. DTW can handle sequences with different lengths and local time shifting, but it does not follow triangle inequality, which is one of important properties of a metric distance function. Since most of the indexing structures assume that the underlying distance functions follow triangle inequality, DTW is non-indexable with current techniques. ERP can support local time shifting and is a metric distance function. LCSS is proposed to handle possible noise that may appear in data; however, it ignores various gaps in between similar subsequences, which leads to inaccuracy.

Not much work has been done in a similar vein for monitoring stream time series data. Zhu and Shasha [34] proposed a method to monitor the correlation among any pair of stream time series data within a sliding window, in which DFT was used as a summary of the data. Later, they introduced a shift wavelet tree (SWT) based on DWT to monitor bursts over stream time series data [33]. Bulut and Singh [6] improved the technique by using multi-scaled DWT trees to summarize the data. Gao and Wang [13] proposed a prediction model to save the computation cost during the matching between a single stream time series to multiple static patterns. Previous works all focused on either single pattern or single stream, our work, however, considers multiple static patterns and multiple time series from streams. Recently, Papadimitriou et al. [22] proposed a method for capturing correlations among multiple stream time series data with the help of an incremental PCA computation method. With respect to data estimation, $\mathrm{Yi}$ et al. [32] estimated the current values of a coevolving time series through a multi-variant linear 
regression. These approaches, however, are different from our similarity match problem, in the sense that they do not assume any query patterns available. Instead, they aim at detecting either patterns in a stream time series or changes in correlation pattern over multiple stream time series.

\section{Problem Statement}

A time-series stream is an ordered sequence, $S=\left(s_{1}\right.$, $\left.s_{2}, \ldots, s_{i}, \ldots, s_{t}\right)$, where each $s_{i}$ is a real value arriving at a specific time $i$, and $t$ the current timestamp. In this paper, we focus on a sliding window model due to its popularity and generality. A sliding window is denoted as $W_{i}=\left(s_{i}, s_{i+1}, \ldots, s_{i+w-1}\right)$, where $w$ is the pre-defined window size. A sliding window $W_{i}$, for $i=1, \ldots$, can be also considered as a $w$-dimensional data.

Assume we have a set of pre-defined time-series patterns, $P=\left\{p_{1}, p_{2}, \ldots, p_{m}\right\}$, where the length of each pattern $p_{i}$ can be greater than or equal to $w$. As illustrated in Definition 1, we study the problem of retrieving the similar match between every window $W_{i}$, for $i=1, \ldots$, in high speed time-series stream $S$ and patterns $p_{i} \in P$. A window $W_{i}$ is similar to a pattern $p_{j}$ if $\operatorname{dist}\left(W_{i}, p_{j}\right) \leq \varepsilon$, where dist is a distance function and $\varepsilon$ a user-specified threshold. Among all possible distance functions for time-series data [9], such as $\mathrm{L}_{p}$-norm, DTW [4], LCSS [27], and ERP [9], in this paper, we use the $\mathrm{L}_{p}$-norm (for any $p \geq 1$ ), which covers a wide range of applications [31]. Formally, the $\mathrm{L}_{p}$-norm of two sequences $X$ and $Y$ of length $n$ is formally denoted as:

$$
L_{p}(X, Y)=\sqrt[p]{\sum_{i=0}^{n-1}|X[i]-Y[i]|^{p}}
$$

where $p \geq 1$. Note that, the commonly-used $\mathrm{L}_{1}$-norm is called the Manhattan distance, whereas $\mathrm{L}_{2}$-norm the Euclidean distance. When $p$ is infinite, $\mathrm{L}_{\circ}-$ norm is defined as:

$$
L_{\infty}(X, Y)=\max _{i=0}^{n-1}\{|X[i]-Y[i]|\} .
$$

Several remarks are given below. First, in time-series streams, time series arrive at high speed, and therefore we need to process queries very fast. Second, although we defined our problem over multiple time-series streams $S$ in Definition 1, the problem of detecting multiple patterns over multiple streams can be essentially induced to that over a single stream. Third, there is a set $P$ containing multiple patterns. We assume these patterns are static. However, due to the efficient index for patterns, our approach can be easily generalized to the dynamic case where patterns can be updated by either inserting new ones or deleting out-ofdated ones.

Given a set of pre-defined patterns, one of the important issues in detecting similar patterns over a stream time series is to reduce the processing cost during the detection. There are some possible but infeasible solutions. First, we build an index, such as Rtree [14], over the pattern set $P$. With the constructed Rtree, the similarity search can be performed by retrieving a set of patterns in R-tree that match an incoming $W_{i}$. However, due to the high dimensionality of time-series data, the efficiency of searching an index with the dimensionality higher than 15 is even worse than the linear scan [28]. In addition, updates to the predefined patterns may cause extra cost in R-tree. Second, another possible approach is to reduce the dimensionality of time-series $W_{i}$ and patterns $p_{j}$ using any dimensionality reduction technique such as DFT [12], DWT [8] and Chebyshev Polinomials [7]. In particular, any one of these techniques reduces the dimensionality of both $W_{i}$ and $p_{j}$, and checks the similarity over the reduced dimensional space, as a filter, with the guarantee that no false dismissals are introduced. In the literature, only DFT [17] and DWT [6] have been used for reducing the dimensionality in the stream time series, however, with the limitation that only one pattern is considered. In this paper, we propose a novel multi-scaled approximation followed by a multistep filtering technique, which can efficiently retrieve similarity matches between patterns and high speed time-series streams.

\section{Multi-Scaled Approximate Pruning}

In this section, we propose multi-scaled approximate pruning to find similarity matches by reducing the search space as well as the computation cost. Specifically, assume there are two time series, $W_{1}$ and $W_{2}$. By "approximate", we mean that there is an approximation of $W_{k}$, denoted as $A\left(W_{k}\right)$, so that it holds that $\operatorname{dist}\left(W_{1}, W_{2}\right)>\varepsilon$, as long as $\operatorname{dist}\left(A\left(W_{1}\right), A\left(W_{2}\right)\right)>\varepsilon$. In addition, we are looking for a multi-scaled approximation with any $\mathrm{L}_{p}$-norm, such that if it holds that $\operatorname{dist}\left(A_{j}\left(W_{1}\right), A_{j}\left(W_{2}\right)\right)>\varepsilon$, then $\operatorname{dist}\left(W_{1}, W_{2}\right)>\varepsilon$, where $A_{j}\left(W_{k}\right)$ is an approximation of time series $W_{k}$ on level $j$. For any two levels $i$ and $j$ where $i<j$, the approximation $A_{j}\left(W_{k}\right)$ at a higher level means a finer approximation. In other words, the approximation on level $j$ gives more information about a time series than the one on level $i$.

In the sequel, Section 4.1 proposes a novel multiscaled approximation, MSM, for any $\mathrm{L}_{p}$-norm with the advantage of the incremental computation. Section 4.2 presents the pruning approach based on the proposed representation. Section 4.3 discusses the similarity match in detail. Section 4.4 illustrates the existing multi-scaled approximation, wavelets (DWT), which is however only applicable to $\mathrm{L}_{2}$-norm, rather than other $\mathrm{L}_{p}$-norm in general, and proves that our MSM approximation has the same pruning power as the 
wavelet-based approach under $\mathrm{L}_{2}$-norm, however higher under other $\mathrm{L}_{p}$-norms.

\subsection{Multi-Scaled Segment-Mean Approximation}

In this section, we propose a novel multi-scaled segment mean approximation (MSM) which is based on the segment mean $[16,31]$. Specifically, suppose we have a time series $W$ of length $w$, where $w=2^{l}$ for a non-negative $l^{1}$. We construct MSM approximation of $W$ by computing the segment mean representations from level 1 (most coarse) to level $l$ (finest). In particular, on each level $i$, there are totally $2^{i-1}$ disjoined segments of equal size $2^{l-i+1}$, each of which is represented by the mean of all values within itself. Figure 1 illustrates a simple example of MSM representation of a time series $W$ with length $w=16$ and thus $l=4$. At the approximation scale 4 , there are in total 8 segments, each of which contains the mean of 2 values within itself. Similarly, on level 3 there are 4 segments, each of which is represented by the average of 4 values which are concatenated by two adjacent segments on level 4 .

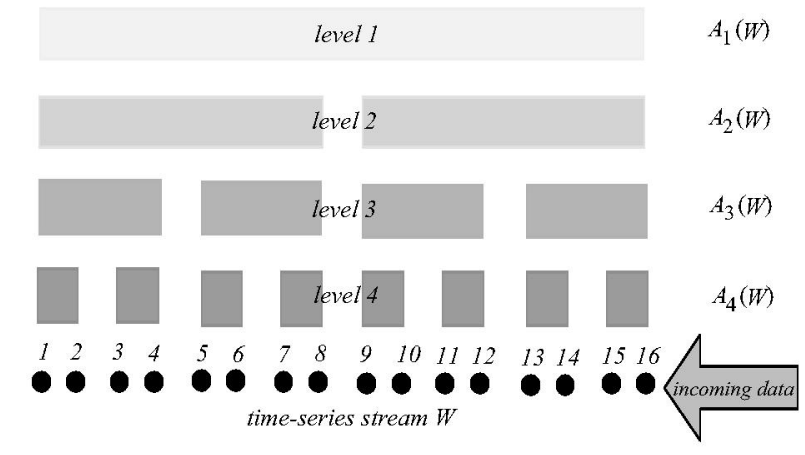

Figure 1: Illustration of the MSM Approximation

Formally, the MSM approximation $A(W)$ for $W$ is given as follows.

$$
A(W)=\left[A_{1}(W), A_{2}(W), \ldots, A_{l}(W)\right]
$$

where $A_{j}(W)$ is a segment mean approximation for $W$ on level $j$ for $1 \leq j \leq l$, and

$$
A_{j}(W)=\left[\mu_{1, j}, \mu_{2, j}, \ldots, \mu_{2^{j}, j}\right]
$$

where $\mu_{i, j}$ is the mean of values $m_{i, j}$ within the $i$-th segment in $W$ on level $j$, for $j=1$ to $l$ and $i=1$ to $2^{j}$.

Note that, although the segment mean representation of a time series is well-studied in literature [16,31], no previous work has been proposed to approximate the time series with a multi-scaled representation. Later on we will show the effectiveness and efficiency of such representation during the similarity match for stream processing.

Remark 4.1 Mean is not an additive function. However, since the number of values in a segment on level $j$ is

\footnotetext{
${ }^{1}$ Note that, here we assume the length $w$ of $W$ is a multiple of 2; otherwise, a sequence of 0 can be appended $[14,32]$.
}

pre-determined as $2^{l-j+1}$, we can incrementally maintain the sum in a segment, $\left(\sigma_{i, j}=\Sigma_{s k \in m_{i j}}\left(s_{k}\right)\right)$, for $m_{i, j}$, and efficiently compute the mean, $\mu_{i, j}$, as $\sigma_{i, j} / 2^{l-j+1}$, when needed. Furthermore, the mean on level $j$ can be computed from the mean on level $j+1$, and treated as an additive function.

Theorem 4.1 Given two time series of length $w\left(=2^{l}\right)$, $W$ and $W^{\prime}$, it holds that

$$
2^{1 / p} \cdot L_{p}\left(A_{i}(W), A_{i}\left(W^{\prime}\right)\right) \leq L_{p}\left(A_{i+1}(W), A_{i+1}\left(W^{\prime}\right)\right)
$$

where $1 \leq i<l$.

Proof sketch: We show our proof, based on two previous results in [24, 31]. First, in [18], Korn et al. showed that

$$
f\left(\lambda_{1} x_{1}+\ldots+\lambda_{w} x_{w}\right) \leq \lambda_{1} f\left(x_{1}\right)+\ldots+\lambda_{w} f\left(x_{w}\right)
$$

where $f\left(x_{i}\right)$ is a convex function on real values $x_{i}$, and $\lambda_{1}, \ldots, \lambda_{w}$ are non-negative real values such that $\sum_{i=1}^{w} \lambda_{i}$ $=1$. Second, based on Eq. (6), Yi and Faloutsos [31] showed that for a sequence $W=\left[s_{1}, \ldots, s_{w}\right]$ and $p \geq 1$,

$$
w \cdot|\operatorname{mean}(W)|^{p} \leq \sum_{i=1}^{w}\left|s_{i}\right|^{p}
$$

where mean $(W)$ is the mean of all values $s_{i} \in W$. Eq. (7) holds for any $\mathrm{L}_{p}$-norm which is convex function on the real set (i.e. $p \geq 1$ ). Briefly, let $f(x)=|x|^{p}$, and $\lambda_{i}=$ $1 / w$. Then $f\left(\sum_{i=1}^{w} \lambda_{i} s_{i}\right)=f\left(\left(\sum_{i=1}^{w} s_{i} / w\right)=\mid\right.$ mean $\left.(W)\right|^{p}$.

Based on Eq. (6) and Eq. (7), we give our proof of Theorem 4.1 for $\mathrm{L}_{p}$-norm where $p=1$, by induction. The same proof can be obtained for arbitrary $\mathrm{L}_{p}$-norm.

Base-case: for an $l=2$, we show that

$$
2 \cdot \mathrm{L}_{1}\left(A_{1}(W), A_{1}\left(W^{\prime}\right)\right)<\mathrm{L}_{1}\left(A_{2}(W), A_{2}\left(W^{\prime}\right)\right) \text { holds. }
$$

Here, based on the definition and Remark 4.1, we have

$$
\begin{aligned}
& A_{1}(W)=\mu_{1,1}=\frac{\mu_{1,2}+\mu_{2,2}}{2} \\
& A_{1}\left(W^{\prime}\right)=\mu_{1,1}^{\prime}=\frac{\mu_{1,2}^{\prime}+\mu_{2,2}^{\prime}}{2}
\end{aligned}
$$

Based on Eq. (9), we have

$$
2 \cdot\left(\left|A_{1}(W)-A_{1}\left(W^{\prime}\right)\right|\right) \leq \sum_{i=1}^{2}\left|\mu_{i, 2}-\mu_{i, 2}^{\prime}\right|
$$

General-Case: we show Theorem 4.1 holds for any level $j$ in a total $l$ level approximation. Eq. (7) holds for $i=1$ when $l=2$ (two levels). Based on this, it holds for every segment $m_{i, j}$ for $1 \leq j<l$, because every segment $m_{i, j}$, at level $j$, consists of two segments at level $j+1$.

$$
\begin{aligned}
& \mu_{i, j}=\frac{1}{2}\left(\mu_{2 i-1, j+1}+\mu_{2 i, j+1}\right) \\
& \mu_{i, j}^{\prime}=\frac{1}{2}\left(\mu_{2 i-1, j+1}^{\prime}+\mu_{2 i, j+1}^{\prime}\right)
\end{aligned}
$$

That is, Eq. (7) holds for every segment $m_{i, j}$ on level $j$. Given an $l$-scaled approximation, for any level $j$, for $1 \leq$ $j<l$, we have

$$
L_{1}\left(A_{j}(W), A_{j}\left(W^{\prime}\right)\right)=\sum_{i=1}^{2^{\prime-1}}\left|\mu_{i, j}-\mu_{i, j}^{\prime}\right|
$$




$$
\begin{aligned}
& \leq \frac{1}{2} \sum_{i=1}^{2^{\prime}}\left(\left|\mu_{2 i-1, j+1}-\mu_{2 i-1, j+1}^{\prime}\right|+\left|\mu_{2 i, j+1}-\mu_{2 i, j+1}^{\prime}\right|\right) \\
& =\frac{1}{2} \mathrm{~L}_{1}\left(A_{j+1}(W), A_{j+1}\left(W^{\prime}\right)\right)
\end{aligned}
$$

Therefore, Eq. (7) holds for any level $j$.

Since the time-series $W=\left[s_{1}, s_{2}, \ldots, s_{w}\right]$ itself, for $w=2^{l}$, can be considered as a special approximation at level $\log _{2}(w)+1$, we have the following corollary.

Corollary 4.1 Given two time series $W$ and $W^{\prime}$ of length $w\left(=2^{l}\right)$, we have

$$
2^{\frac{\log _{2}(w)+1-j}{p}} L_{p}\left(A_{j}(W), A_{j}\left(W^{\prime}\right) \leq L_{p}\left(W, W^{\prime}\right)\right.
$$

\subsection{Pruning with MSM Approximation}

In this section, we present a simple yet effective pruning algorithm, namely segment mean pruning $(S M P)$, as illustrated in Algorithm 1. For a given window size $w\left(=2^{l}\right)$, Algorithm 1 takes four parameters, $P, W, \mathcal{E}$, and $G I$, where $P$ is a set of patterns, $W$ a window of an incoming time series with size $w, \varepsilon$ a similarity threshold and $G I$ a multidimensional grid indexing pattern ids in $P$. Algorithm 1 returns a subset $P^{\prime}$ of the pattern set $P$ that may match with $W$. As a result, each candidate pattern $p \in P^{\prime}$ can be further refined by checking the actual distance between $W$ and $p$.

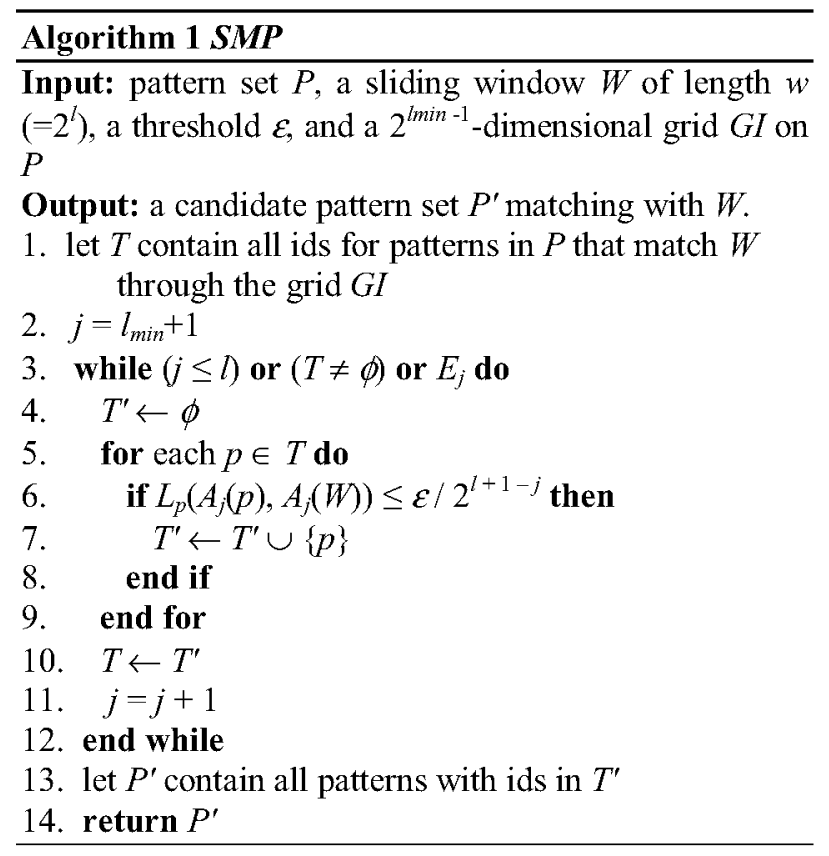

Without loss of generality, assume that we have already calculated MSM approximations $A_{j}(p)$ on any level $j$ where $1 \leq j \leq l$, for each pattern $p$ in $P$ and materialized them. Furthermore, suppose the grid index $G I$ is of $2^{l m i n-1}$ dimensions, which is usually low (the typical value is 1 or 2), where $1 \leq l_{\min } \leq l$. Each cell of the index $G I$ contains pattern identifies with their approximations on level $l_{\min }$ falling into it. We briefly illustrate each step of Algorithm 1 as follows. First, we access the grid index $G I$ to obtain all pattern identifies that have distances from the MSM approximation $A_{\text {Imin }}(W)$ within $\varepsilon$ on level $l_{\min }$. Then, we initialize a set $T$ containing identifiers of the result (line 1) and $j=l_{\min }+1$ (line 2). The pruning procedure repeats in a whilestatement with approximations at different scales from $j$ $=l_{\min }+1$ to $j=l$ (line 3-12). There are three conditions for a while-statement to terminate (line 3): (i) $j$ reaches the last approximation level $l$, (2) there are no matching patterns left, or (3) an early stop condition, $E_{j}$, is satisfied, which we discuss later in this section. In each iteration $j$, there is a for-statement to check whether or not each pattern $p$ in $T$ satisfies Eq. (11) (line 6). If it is met, $p$ is added to $T^{\prime \prime}$ (line 7), which is then assigned to $T$ before the next iteration $j+1$. Finally, we return $P^{\prime}$ corresponding to identifiers in $T^{\prime}$ that may match with $W$ (lines 13-14).

Next, we discuss the early stop condition $E_{j}$ to stop pruning when $j<l$. Note that there are total $|P|$ patterns. Let $P_{j}$ be the percentage of unpruned objects, in terms of the total number $N$ of data objects in a time series, after pruning at the level $j$. We have $P_{i} \geq P_{j}$ for $i<j$. Let $P_{\text {lmin }}$ be the percentage after searching in the grid index. Then, assume the average computation cost of computing distance between two objects is $C_{d}$ and we stop pruning at level $j(\leq l)$. Therefore, the total cost is

$$
\cos t_{j}=\sum_{i=l_{\min }}^{j-1}\left(N \cdot P_{i} \cdot|P| \cdot 2^{i} \cdot C_{d}\right)+N \cdot P_{j} \cdot|P| \cdot w \cdot C_{d}
$$

Here, the first term is the total cost of computing approximation. Considering level $j-1$, there are $N \cdot P_{j-1}$ objects left before pruning. Then, we need to compute distance for each object from $|P|$ patterns using $2^{j-1}$ segments and obtain candidates at level $j$. The second term is the cost to compute similarity between $|P|$ patterns and the unpruned $N \cdot P_{j}$ windows. It is worth noting that we can use the scale $j$ to do the further filtering only if

$$
\operatorname{cost}_{j-1} \geq \cos t_{j} \text {. }
$$

Furthermore, based on Eq. (12) and Eq. (13), we have

$$
\log \frac{P_{j-1}-P_{j}}{P_{j-1}} \geq(j-1-\log (w)) .
$$

It is important to know that we can continue pruning on level $j$, if Eq. (14) holds, which is the early stop condition we use as $E_{j}$ in Algorithm 1.

Eq. (14) gives the cost analysis of our pruning scheme, where $j$ is the early abort level depending on three stopping conditions in line 3 of Algorithm 1. As evaluated by our experiments, $j$ is usually much smaller than $l(\log w)$, and therefore the filtering scheme is efficient by comparing only a few levels of approximations. 
Discussion on Pruning Schemes. Besides our pruning approach (denoted as step-by-step, or SS) which prunes objects with approximations level by level, we have also considered other schemes, including jump-step (JS) and one-step (OS) schemes. As we discuss further on, SS is the best one among the three as long as certain conditions hold. In the sequel, we denote cost $\operatorname{cost}_{j}$ in Eq. (12) of SS as cost $t_{\mathrm{SS}}$.

First, we consider JS scheme which only chooses two levels $\left(l_{\min }+1\right)$ and $j$. The computational cost $\operatorname{cost}_{\mathrm{JS}}$ of this scheme is as follows:

$$
\begin{aligned}
\operatorname{cost}_{\mathrm{JS}}= & N \cdot P_{l_{\min }} \cdot|P| \cdot 2^{l_{\min }} \cdot C_{d}+N \cdot P_{l_{\min }+1} \cdot|P| \cdot 2^{j-1} \cdot C_{d} \\
& +N \cdot P_{j} \cdot|P| \cdot w \cdot C_{d}
\end{aligned}
$$

Compared with SS scheme, Eq. (15) immediately gives the following theorem:

Theorem 4.2 Given two schemes JS and SS, as long as $P_{\text {Imin }+1} \geq 2 \cdot P_{\text {lmin }+2}$ holds, cost $t_{\mathrm{SS}} \leq \operatorname{cost}_{\mathrm{JS}}$ also holds.

Proof sketch: Based on Eq. (15) and Eq. (12), we have: $\operatorname{cost}_{\mathrm{SS}}-O s t_{\mathrm{JS}}$

$$
\begin{aligned}
= & N \cdot|P| \cdot C_{d} \cdot\left(\left(\sum_{i=l_{\min }}^{j-1}\left(P_{i} \cdot 2^{i}\right)+P_{j} \cdot w\right)\right. \\
& \left.-\left(P_{l_{\min }} \cdot 2^{l_{\min }}+P_{l_{\min }+1} \cdot 2^{j-1}+P_{j} \cdot w\right)\right) \\
= & N \cdot|P| \cdot C_{d} \cdot\left(\sum_{i=l_{\min }+1}^{j-1}\left(P_{i} \cdot 2^{i}\right)-P_{l_{\min }+1} \cdot 2^{j-1}\right) .
\end{aligned}
$$

Since $P_{l_{\min }+2} \geq P_{i}\left(i \geq l_{\min }+2\right)$, we replace all such $P_{i}(i \geq$ $l_{\text {min }}+2$ ) with $P_{\text {lmin }+2}$ in Eq. (16), thus having

$$
\begin{aligned}
& \cos t_{\mathrm{SS}}-\cos _{\mathrm{JS}} \\
\leq & N \cdot|P| \cdot C_{d} \cdot\left(P_{l_{\min }+1} \cdot\left(2^{l_{\min }+1}-2^{j-1}\right)+P_{l_{\min }+2} \cdot \sum_{i=l_{\min }+2}^{j-1} 2^{i}\right) \\
= & N \cdot|P| \cdot C_{d} \cdot\left(P_{l_{\min }+1} \cdot\left(2^{l_{\min }+1}-2^{j-1}\right)-P_{l_{\min }+2} \cdot\left(2^{l_{\min }+2}-2^{j}\right)\right) .
\end{aligned}
$$

Assuming the right hand side of Eq (17) is non-positive, that is,

$$
N \cdot|P| \cdot C_{d} \cdot\left(P_{l_{\min }+1} \cdot\left(2^{l_{\min }+1}-2^{j-1}\right)-P_{l_{\min }+2} \cdot\left(2^{l_{\min }+2}-2^{j}\right)\right) \leq 0,
$$

we obtain exactly $P_{l \min +1} \geq 2 \cdot P_{l \min +2}$. In other words, $P_{\text {lmin }+1} \geq 2 \cdot P_{\text {lmin }+2}$ implies $\cos t_{\mathrm{SS}}-\cos _{\mathrm{JS}} \leq 0$, which completes the proof.

As a second step, we discuss OS scheme which uses only one level $j$ for pruning. The computational cost cost $_{\mathrm{OS}}$ of OS is given by:

$$
\operatorname{cost}_{\mathrm{OS}}=N \cdot P_{l_{\min }} \cdot|P| \cdot 2^{j-1} \cdot C_{d}+N \cdot P_{j} \cdot|P| \cdot w \cdot C_{d} \cdot
$$

Theorem 4.3 Given two schemes OS and SS, as long as $P_{l m i n} \geq 2 \cdot P_{l m i n+1}$ holds, cost $t_{\mathrm{SS}} \leq$ cost $t_{\mathrm{OS}}$ also holds.

Proof sketch: Similar to the proof of Theorem 4.4, based on Eq. (19) and Eq. (12), we have

$\operatorname{cost}_{\mathrm{SS}}-\operatorname{cost}_{\mathrm{OS}}$

$$
=N \cdot|P| \cdot C_{d} \cdot\left(\left(\sum_{i=l_{\min }}^{j-1}\left(P_{i} \cdot 2^{i}\right)+P_{j} \cdot w\right)-\left(P_{l_{\min }} \cdot 2^{j-1}+P_{j} \cdot w\right)\right)
$$

$$
=N \cdot|P| \cdot C_{d} \cdot\left(\sum_{i=l_{\min }}^{j-1}\left(P_{i} \cdot 2^{i}\right)+P_{l_{\min }} \cdot\left(2^{l_{\min }}-2^{j-1}\right)\right) .
$$

Since $P_{\text {lmin }+1} \geq P_{i}\left(i \geq l_{\text {min }}+1\right)$, we replace all such $P_{i}(i \geq$ $l_{\text {min }}+1$ ) with $P_{\text {lmin }+1}$ in Eq. (20), obtaining

$$
\cos _{\mathrm{SS}}-\cos _{\mathrm{OS}}
$$

$\leq N \cdot|P| \cdot C_{d} \cdot\left(P_{l_{\min }+1} \cdot \sum_{i=l_{\min }+1}^{j-1} 2^{i}+P_{l_{\min }} \cdot\left(2^{l_{\min }}-2^{j-1}\right)\right)$

$=N \cdot|P| \cdot C_{d} \cdot\left(P_{l_{\min }+1} \cdot\left(2^{l_{\min }+1}-2^{j}\right)+P_{l_{\min }} \cdot\left(2^{l_{\min }}-2^{j-1}\right)\right)$

If the right hand side of Eq. (21) is not greater than zero, then it holds that

$$
N \cdot|P| \cdot C_{d} \cdot\left(P_{l_{\min }+1} \cdot\left(2^{l_{\min }+1}-2^{j}\right)+P_{l_{\min }} \cdot\left(2^{l_{\min }}-2^{j-1}\right)\right) \leq 0,
$$

which implies $P_{l m i n} \geq 2 \cdot P_{l m i n+1}$. Therefore, as long as Eq. (22) holds, $\cos t_{\mathrm{SS}}-$ cost $_{\mathrm{OS}} \leq 0$ also holds.

In summary, from Theorems 4.2 and 4.3 , we can see that at level $l_{\min }+1$, as long as the approximation can filter out more than $50 \%$ of the objects, SS has lower cost than OS. Furthermore, if the level $\left(l_{\min }+2\right)$ approximations have the ability to filter out at least $50 \%$ of the objects left from the level $\left(l_{\min }+1\right)$ pruning, SS outperforms JS. By investigating extensive experiments, we would always select SS as our multi-scaled pruning scheme in the sequel. Finally, since the SS pruning scheme only uses approximations up to level $l_{\max }$, the space requirement of each sliding window $W$ is $2^{l_{\max }-1}$. That is, it is enough to store approximation $A_{\text {lmax }-1}(W)$ on level $\left(l_{\max }-1\right)$, from which lower levels can be computed.

\subsection{Similarity Match}

Based on MSM approximation discussed above, Algorithm 2 Similarity_Match illustrates the procedure to retrieve the segment-mean similarity match between patterns and a stream time series $S$. Specifically, in order to speed up pruning with MSM, we create a $2^{l_{\min }-1}$ dimension grid-based index $G I$ for level $l_{\text {min }}$ approximation of patterns, where $2^{l_{\min }-1}$ is typically low (e.g. 1 or 2). As an example, assuming $l_{\min }=1$, we use a 1-dimension grid $G I$ to index patterns in $P$, where the size of each cell is set to $\varepsilon$. Inside each cell, we keep those patterns having the mean values $\mu_{1,1}$ within the cell range. Similarly, in the case of $l_{\min }=2$, we construct a 2-dimension grid containing cells with size $\mathcal{E} / \sqrt{2}$ and index patterns in $P$ with pairs of two mean values $\mu_{1,2}$ and $\mu_{2,2}$ in it. Note that, although we consider the grid of equal size here, it can be easily extended to that of skewed sizes that are adaptive to the mean distribution of patterns.

In Algorithm 2, we invoke Algorithm $1 S M P$ to find all patterns $P^{\prime}$ in $P$ that are matched against the latest sliding windows $W_{i}$ in a time series $S$ (lines 2-3). Then, for each candidate pattern $p \in P^{\prime}$, we calculate the real 
distance between $p$ and $W_{i}$, and finally output the pair $\left(W_{i}, p\right)$ if they are similar (lines 4-8).

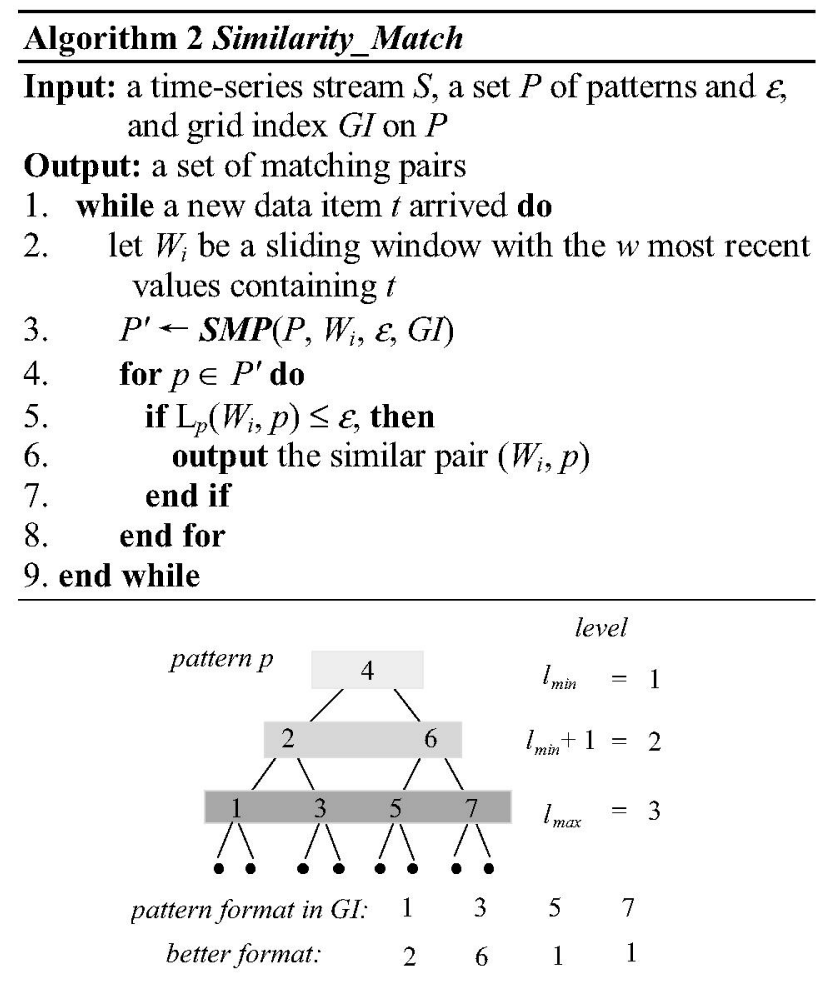

Figure 2: Illustration of Pattern Representation

Note that, here each pattern $p$ in the grid index $G I$ is represented by $2^{l_{\max }-1}$ values at scale $l_{\max }$ in the form $A_{l \max }(p)$, where $l_{\max }$ is upper-bounded by the value of $j$ such that Eq. (14) does not hold. As an example in Figure 2, a pattern $p$ is with an MSM representation from level $l_{\min }$ to $l_{\max }$, assuming $l_{\min }=1$ and $l_{\max }=3$. In the grid index $G I$, we store the level 3 approximation $A_{3}(p)$ (i.e. on level $l_{\max }$ ), since both $A_{1}(p)$ and $A_{2}(p)$ on levels 1 and 2 , respectively, can be calculated from $A_{3}(p)$. That is, $A_{2}(p)=<(1+3) / 2,(5+7) / 2>$ and $A_{1}(p)=$ $<(1+3+5+7) / 4>$.

However, since the procedure applies SS scheme (line 3 in Algorithm 2), we need to perform the matching test with approximations of $p$ from level $\left(l_{\min }+1\right)$ to $l_{\max }$, where each level has to be calculated from $A_{\text {lmax }}(p)$. This incurs high computational cost, and thus optimizations need to be carried out. In particular, for any pattern $p$, we record its level $\left(l_{\text {min }}+1\right)$ approximation $A_{l m i n+1}(p)$ in $G I$. In addition, similar to DWT, we also store differences of level $\left(l_{\min }+2\right)$ from level $\left(l_{\text {min }}+1\right)$, level $\left(l_{\text {min }}+3\right)$ from level $\left(l_{\text {min }}+2\right)$, and so on. As in the example, we keep the pattern in the form $<2,6,1,1>$ in $G I$, where the first two values " 2 " and " 6 " are on level $2\left(=l_{\min }+1\right)$, the third " 1 " is the difference between " 3 " and " 2 " on levels 3 and 2, respectively, and the second " 1 " is that between "7" and "6" also on levels 3 and 2, respectively. The advantage of this representation is that, approximation of patterns on the next level can be always calculated with low cost using differences, and computation cost can be saved if SS scheme aborts early. In summary, the space requirement of grid $G I$ is given by $2^{l_{\max }-1} \cdot|P|$, where $l_{\max }$ is the maximum possible level used in SS, and $|P|$ the number of patterns.

\subsection{Multi-Scaled Wavelet v.s. Segment-Mean}

In this section, we compare the MSM approximation with another multi-scaled representation, Discrete Wavelet Transform (DWT), which has been widely used for the image retrieval [20] as well as a dimensionality reduction technique $[8,23]$. In particular, DWT can also transform the time series data to a multi-scaled representation, where lower frequency bands are represented as lower scales and higher frequency bands are recorded as higher scales. One of the most popular wavelets is the Haar wavelet, which has been widely used in image [26], speech [4], and signal processing [3], since it can be quickly computed with a linear cost. Chan and $\mathrm{Fu}$ [8] proved that the $\mathrm{L}_{2}$-norm distance between the first few Haar wavelet coefficients is the lower bound of that between the original time series. A theorem in [8] is given as follows.

Theorem 4.4 ([8]) Let $W$ and $W^{\prime}$ be two time-series with the same length $w\left(=2^{l}\right)$, and $H(W)$ and $H\left(W^{\prime}\right)$ be the Haar Transforms of $W$ and $W^{\prime}$, respectively. Let $H(W)-$ $H\left(W^{\prime}\right)=\left[c, d_{1}, \ldots, d_{w-1}\right]$. The Euclidean distance $L_{2-}$ norm $(W, W)$ can be recursively computed from $[c$, $\left.d_{1}, \ldots, d_{w-1}\right]$ as follows:

$$
\begin{aligned}
& \delta_{0}=c \\
& \delta_{i+1}=\sqrt{\left(\delta_{i}^{2}+d_{2^{i}}^{2}+d_{2^{i}+1}^{2}+\ldots++d_{2^{i+1}-1}^{2}\right)}
\end{aligned}
$$

for $0 \leq i \leq \log _{2} w-1$.

According to Theorem 4.4, we can construct a multiscaled representation of a time series by taking different numbers of coefficients of the transformed Haar wavelet at each scale. In particular, we take the first $2^{i-1}$ coefficients of the wavelet for each scale $i$, where $1 \leq i$ $\leq \log _{2} w$. Based on Theorem 4.4, we have the following corollary that claims the $\mathrm{L}_{2}$-norm computed at a scale $i$ is the lower bound of that computed at the scale $j$ satisfying $i \leq j$.

Corollary 4.2 Given two levels $i$ and $j$, wavelet coefficients $(i \leq j)$, the $L_{2}$-norm computed at scale $i$ is the lower bound of $L_{2}$-norm computed in scale $j$.

It is worth noting that since DWT is an orthogonal linear transformation, only $\mathrm{L}_{2}$-norm is preserved under this transformation. In the following theorem, we show that the pruning power of $\mathrm{MSM} \mathrm{L}_{2}$-norm is the same as that of DWT.

Theorem 4.5 Given a time series of $W=\left(s_{1}, s_{2}, \ldots, s_{w}\right)$ of length $w=2^{l}$, and let its multi-scaled wavelet approximation be $H(W)=\left[h_{1}, h_{2}, \ldots, h_{w}\right]$ where $h_{j}=$ 
$\left[h_{1, j}, h_{2, j}, \ldots, h_{\left.2^{j}, j\right]}\right.$ and its multi-scaled segment-mean approximation be $A(W)=\left[\mu_{1}, \mu_{2}, \ldots, \mu_{w}\right]$ where $\mu_{j}=$ $\left[\mu_{1, j}, \mu_{2, j ;}, \ldots, \mu_{2^{j}}, j\right]$. For any level $1 \leq j \leq l$, we have $\left|h_{j}\right|^{2}$ $=2^{i+1-j}\left|\mu_{j}\right|^{2}$.

Proof : By induction, for the base case, it is true for $j=$ 1 , such as $\left|h_{1}\right|^{2}=2^{l} \cdot\left|\mu_{1}\right|^{2}$, since

$$
\begin{aligned}
& \left|h_{1}\right|^{2}=\left|\frac{\operatorname{sum}(W)}{(\sqrt{2})^{l}}\right|^{2}=\frac{\operatorname{sum}(W)^{2}}{w}, \\
& 2^{l} \cdot\left|\mu_{1}\right|^{2}=w \cdot|\operatorname{mean}(W)|^{2}=\frac{\operatorname{sum}(W)^{2}}{w} .
\end{aligned}
$$

Assume the claim holds for $j>1$ that $\left|h_{j}\right|^{2}=2^{l+1-j}\left|\mu_{j}\right|^{2}$. We show that, for $j+1$, it holds that

$$
\begin{aligned}
& 2^{l+1-(j+1)}\left|\mu_{j+1}\right|^{2}-2^{l+1-j}\left|\mu_{j}\right|^{2} \\
&= 2^{l+1-j}\left(\frac{1}{2} \mu_{j+1}^{2}-\mu_{j}^{2}\right) \\
&= 2^{l+1-j}\left(\frac{1}{2}\left(\mu_{1, j+1}^{2}+\ldots+\mu_{2^{j+1}, j+1}^{2}\right)-\left(\mu_{1, j}^{2}+\ldots+\mu_{2^{j}, j}^{2}\right)\right) \\
&= d_{2^{j}}^{2}+d_{2^{j}+1}^{2}+\ldots+d_{2^{j+1}-1}^{2} \\
&= h^{2}{ }_{j+1}-h_{j}^{2} \\
& \text { Thus } 2^{l+1-(j+1)}\left|\mu_{j+1}\right|^{2}=\left|h_{j+1}\right|^{2} \text { holds. }
\end{aligned}
$$

However, for $\mathrm{L}_{p}$-norm other than $\mathrm{L}_{2}$-norm, the distance of DWT is not preserved at all [31]. Therefore, we cannot make use of DWT under $\mathrm{L}_{p}$-norms except for $\mathrm{L}_{2}$-norm [31], whereas MSM can work under arbitrary $\mathrm{L}_{p}$-norm. Furthermore, the computational cost of DWT is higher than that of the MSM approximation. This is because for an $l$-scaled approximation, MSM needs to compute $2^{l}$ means for segments, whereas in addition to means, DWT needs to compute $2^{l}$ more wavelet coefficients for segments. Thus, MSM is more efficient than DTW and it can be applied to the similarity search under any $L_{p}$-norm.

\section{Experiments}

In this section, we report the empirical study on the effectiveness and efficiency of our multi-scaled approximate pruning. The experimental evaluation consists of two parts. First, we verified that our multistep filtering SS with MSM representation indeed improves the retrieval efficiency, compared to other methods OS and JS. Moreover, we tested analytic results of SS. As a second step, we compared the MSM with DWT representation, in terms of efficiency in detecting patterns. Both real and synthetic data were used in the experiments. Specifically, for the real data, we use 24 benchmark datasets, and a stock data set [19] which contains two years' New York Stock Exchange tick-by-tick real data (year 2001 and 2002). For the synthetic data, both stream and pattern time series data are generated using the random walk model. For stream $S$, an element $s_{i}$ of $S$ is $s_{i}=R+\sum_{j=1}^{i}\left(\mu_{j}-0.5\right)$ where $R$ is a constant real number in $[0,100]$, and $\mu_{j}$ a set of uniform random numbers in $[0,1]$. Since our similarity match over the stream time series happens in memory, we assume that at each timestamp, the last sliding windows of all stream time series can fit in the memory. Thus, we always measure the average computation time (CPU time) for similarity queries, which can also reveal the pruning power and savings of the computation cost. We conducted experiments on a Pentium 4 PC with $512 \mathrm{M}$ memory. All results are averaged over 20 runs.

\subsection{Efficiency Test on Multi-step Filtering Strategy}

In our first experiment, we verified the efficiency of the multi-step filtering SS over 24 benchmark datasets $[15,34,9]$, compared to OS and JS. The tested data represent a wide spectrum of applications and data characteristics, where the length of each time series is 256. We randomly picked up a time series from each dataset and conduct a range query at all scales. Then, we compared three different filtering schemes, SS, JS and OS, using the MSM in $\mathrm{L}_{2}$-norm. Figure 3 shows the experimental result, where the $X$-axis is 24 benchmark datasets and the $Z$-axis is the CPU time. As we expected, MSM representation together with the multi-step filtering performs efficiently (within a few milliseconds) on all the 24 datasets. Specifically, the filtering mechanism SS achieves the best performance, followed by JS and OS. By further investigating the average pruning power of the first scale in a multi-scaled segment mean, we found that the first scale representation indeed filtered out over $50 \%$ of the data in each dataset. Again, by checking the average number of remaining results after the filtering at each scale, we did find that $P_{2}<50 \% P_{1}$ holds. Similar results on the real stock and synthetic randomwalk datasets are obtained with the same settings of experiments, but will not be presented due to the space limit.

In the next experiment, we verified the relationship between the highest "useful" level $j$ and $\log \frac{P_{i-1}-P_{i}}{P_{j-1}}$ based on Eq. (14). In particular, when $(j-1-\log w)>\log \frac{P_{i 1}-P_{i}}{P_{j-1}}$ holds, where $j$ starts from 1 to $l, j$ is considered as the best level for SS to stop filtering. In order to obtain $P_{j}$, we randomly sampled $10 \%$ of the data and calculated the percentage of samples that are left by filtering on level $j$. Table 1 illustrates the CPU time of SS filtering at different scales using four sample benchmark datasets, cstr, soiltemp, sunspot and ballbeam (other 20 datasets work as well). Specifically, for each dataset, we obtain the value of $\log \frac{P_{i-1}-P_{i}}{P_{j-1}}$ on different levels. If $\log \frac{P_{i-1}-P_{i}}{P_{j-1}} \geq j$ $-1-\log w$ ), we highlight the value by bold font. We can see that the maximum scale that the bold font is exactly where SS achieves the best performance (i.e. the lowest CPU time). 


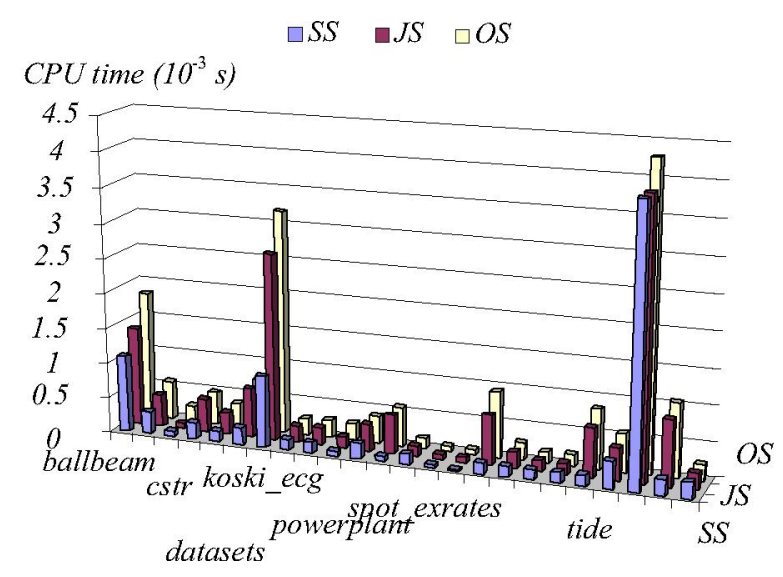

Figure 3: CPU time comparison of different filtering mechanisms 24 benchmark $\left(\mathrm{L}_{2}\right.$-norm)

\begin{tabular}{|c|c|c|c|c|c|c|c|c|c|}
\hline \multirow{3}{*}{ datasets } & \multirow[b]{2}{*}{ measure } & \multicolumn{8}{|c|}{ level (pattern length $=256)$} \\
\hline & & 1 & 2 & 3 & 4 & 5 & 6 & 7 & 8 \\
\hline & $j-1-\log w$ & -8 & -7 & -6 & -5 & -4 & -3 & -2 & -1 \\
\hline \multirow[b]{2}{*}{ cstr } & $\log \frac{P_{i-1}-P_{i}}{P_{j-1}}$ & -0.06 & -0.28 & -2.14 & $\mid-3.02$ & -9.58 & $\mid-5.34$ & -9.47 & -8.54 \\
\hline & \begin{tabular}{|c|} 
CPU time \\
$\left(10^{-4} s\right)$
\end{tabular} & - & 1.15 & 1.55 & 1.1 & 8 & 7.5 & 2.6 & 8 \\
\hline \multirow[t]{2}{*}{ soiltemp } & $\log \frac{P_{j-1}-P_{i}}{P_{j-1}}$ & -0.19 & -0.44 & -0.71 & $\mid-1.52$ & -2.13 & -.3 .38 & -3.87 & -9.62 \\
\hline & $\begin{array}{c}\text { CPU time } \\
\left(10^{-4} \mathrm{~s}\right)\end{array}$ & - & 10.2 & 10.25 & 3.95 & 1.6 & 2.35 & 2.35 & 3.95 \\
\hline \multirow[t]{2}{*}{ sunspot } & $\log \frac{P_{i-1}-P_{i}}{P_{P-1}}$ & -0.13 & -0.37 & -0.64 & -3.27 & -3.92 & -3.7 & -4 & -9.6 \\
\hline & \begin{tabular}{|c|} 
CPU time \\
$\left(10^{-4} \mathrm{~s}\right)$
\end{tabular} & - & 5.5 & 1.6 & 4.7 & 1.55 & 7.5 & 3.15 & 8 \\
\hline \multirow{2}{*}{ ballbeam } & $\log \frac{P_{j-1}-P_{i}}{P_{j-1}}$ & -0.3 & -0.85 & -0.79 & $\mid-0.72$ & -1.43 & -1.6 & -3.51 & -6.34 \\
\hline & \begin{tabular}{|c|} 
CPU time \\
$\left(10^{-4} s\right)$
\end{tabular} & - & 11.75 & 18 & 12.4 & 13.9 & 11 & 23.5 & 22 \\
\hline
\end{tabular}

Table 1: Test the analytic results of multi-step filtering (four sample datasets from 24 benchmark)

\subsection{Performance of Detecting Patterns}

In this section, we report the efficiency test of our MSM representation together with SS filtering in detecting patterns, compared to DWT. Since we consider sliding windows, we perform our experiment on stock and randomwalk datasets, other than 24 benchmark datasets which are only with length 256 . For stock data, we randomly choose 1000 series with length 512 from the generated stock data as patterns, and use the rest as data from stream time series. For randomwalk, we randomly obtain 1000 patterns of length 1024 (512). Specifically, for the stream time series, we consider a sliding window 1.5 times that the length of patterns. That is, we set the sliding window size to 768 for stock data and 1536 (768) for randomwalk. For simplicity, in the remaining experiments, we assume there is a 1-dimensional grid index built for 1000 patterns (i.e. $l_{\min }=1$ ). Therefore, the filtering step always starts from the second level.

For fair comparisons, we use the same level and number of coefficients in both MSM and DWT. Figure 4 illustrates the CPU time of MSM and DWT with 15 stock datasets under different $\mathrm{L}_{p}$-norms. In particular,
Figures 4(a), 4(b), 4(c) and 4(d) illustrate the experimental results with stock data using $\mathrm{L}_{1^{-}}, \mathrm{L}_{2^{-}}, \mathrm{L}_{3}-$ and $\mathrm{L}_{\infty}$-norm, respectively. The evaluated CPU time includes two parts, the cost of incremental updates and similarity search. For $\mathrm{L}_{2}$-norm, DWT representation has a similar performance to our MSM. However, since the update cost of wavelet coefficients is higher than that of ours, DWT is slightly worse than MSM even though we prove that both methods have the same pruning power under $\mathrm{L}_{2}$-norm. Results from $\mathrm{L}_{1}$-norm show that wavelet is not a good method to do filtering under $\mathrm{L}_{1}$-norm. Since $\mathrm{L}_{1}$-norm is not preserved by the wavelet transform, an $\mathrm{L}_{2}$-norm distance has to be computed in order to do the filtering correctly (without introducing false dismissals) [31]. That is the reason that MSM representation is an order of magnitude better than DWT under $\mathrm{L}_{1}$-norm, as shown in Figure 4(a). For $\mathrm{L}_{3}-$ and $\mathrm{L}_{\infty}$-norm, as mentioned in [31], although the wavelet can not preserve $\mathrm{L}_{p}$-norm for $p \neq 2$, the problem can be fixed by issuing range queries under $\mathrm{L}_{2}$-norm with a much larger radius. In particular, for $\mathrm{L}_{3}$-norm, the radius of the range query is set to $\sqrt{3} \cdot \mathcal{E}$, where $\mathcal{E}$ is the original radius; similarly, for $L_{\infty}$-norm, the radius is $\sqrt{w} \cdot \mathcal{E}$, where $w$ is the length of patterns. Note that, in Figure 4(d), the $Z$-axis is in logarithmic scale, indicating that DWT is very inefficient in $\mathrm{L}_{\infty}$-norm. Finally, Figure 5 demonstrates the same set of experiments on synthetic randomwalk dataset, where pattern length is 512 in Figure 5(a) and 1024 in Figure 5(b). The CPU time of DWT is always greater than that of MSM.

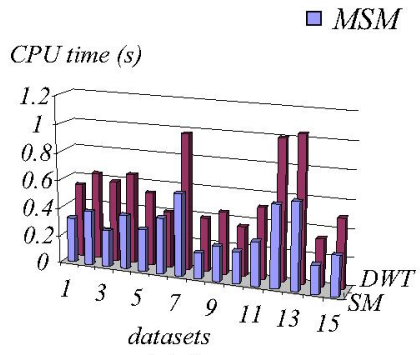

(a) $\mathrm{L}_{1}$-norm

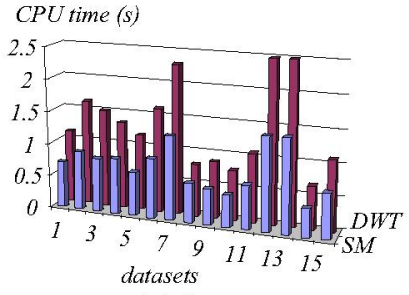

(c) $\mathrm{L}_{3}$-norm

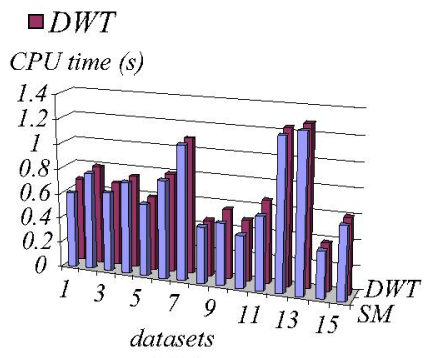

(b) $\mathrm{L}_{2}$-norm

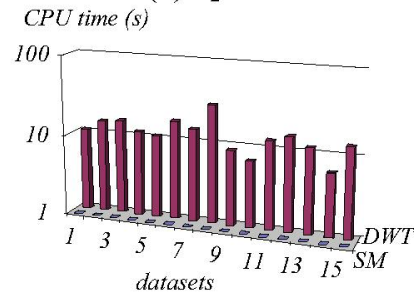

(d) $\mathrm{L}_{\infty}$-norm
Figure 4: Comparison between MSM and DWT (15 stock datasets)

In summary, with 1000 patterns and hundreds of stream data, the MSM representation in all four cases can quickly detect similar patterns over high speed stream time series data for less than a second on average under different $\mathrm{L}_{p}$-norms, compared to DWT. 

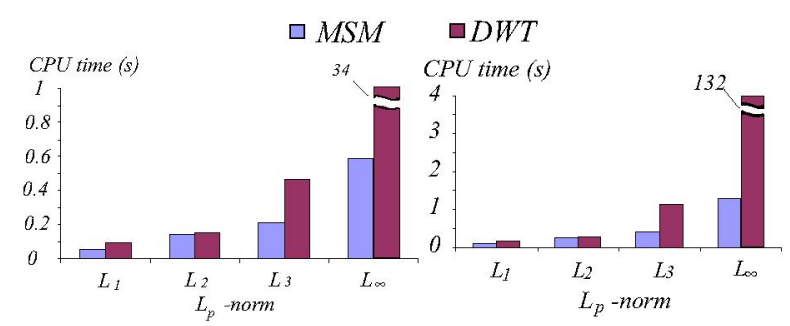

(a) $\mathrm{L}_{p}$ (pattern length $=512$ )

(b) $\mathrm{L}_{p}$ (pattern length $=1024$ )

Figure 5: Comparison between MSM and DWT (randomwalk)

\section{Conclusions}

In this paper, we propose a novel MSM representation together with a multi-step filtering SS scheme, which facilitates detecting patterns over high speed time-series stream efficiently. The MSM representation can be incrementally computed with a low cost and enables us to prune out false alarms with no false dismissals. We show our analytic results on how to derive an optimal aborting level during multistep filtering. Compared to another popular multi-scaled representation, DWT, we prove that MSM has similar computation cost to DWT under $\mathrm{L}_{2}$-norm. However, it is an order of magnitude better under other $\mathrm{L}_{p}$-norms, since MSM is applicable to arbitrary $\mathrm{L}_{p}$-norm, whereas it is not possible for DWT unless a very loose lower bound is used. Extensive experiments show that the multi-step filtering SS on MSM approximations offers an efficient and scalable methodology to detect patterns over high speed stream time series data.

\section{Acknowledgement}

Funding for this work was provided by the Hong Kong RGC grants DAG05/06.EG03, National Basic Research Program of China (2006CB303103), National Natural Science Foundation (60573089), the NSFC Key Project Grant No. 60533110, and National Grand Fundamental Research 973 Program of China under Grant No. 2006CB303000.

\section{References}

[1] R. Agrawal et al. Efficient similarity search in sequence databases. FODO, 1993.

[2] R. Aggrawal et al. Fast similarity search in the presence of noise, scaling, and translation in time-series database. $V L D B, 1995$

[3] A. N. Akansu, P. R. Haddad. Multiresolution signal decomposition. Academic Press, 1992.

[4] D. J. Berndt, J. Clifford. Finding patterns in time series: A dynamic programming approach. In Advances in Knowledge Discovery and Data Mining, 1996.

[5] J. S. Boreczky, L. A. Rowe. Comparison of video shot boundary detection techniques. In Proc. 8th Int. Symp. on Storage and Retrieval for Image and Video Databases, 1996.

[6] A. Bulut, A. K. Singh. A unified framework for monitoring data streams in real time. ICDE, 2005.
[7] Y. Cai, R. Ng. Indexing spatio-temporal trajectories with Chebyshev polynomials. SIGMOD, 2004.

[8] K. P. Chan, A. W. Fu. Efficient time series matching by wavelets. ICDE, 1999.

[9] L. Chen, R. Ng. On the marriage of edit distance and $\mathbf{L}_{p}$ norms. VLDB, 2004.

[10] L. Chen et al. Robust and fast similarity search for moving object trajectories. SIGMOD, 2005.

[11] C. Cranor et al. Gigascope: a stream database for network applications. SIGMOD, 2003.

[12] C. Faloutsos et al. Fast subsequence matching in timeseries databases. SIGMOD, 1994.

[13] L. Gao, X. S. Wang. Continually evaluating similaritybased pattern queries on a streaming time series. SIGMOD, 2002.

[14] A. Guttman. R-trees: a dynamic index structure for spatial searching. SIGMOD, 1984.

[15] E. Keogh. Exact indexing of dynamic time warping. $V L D B, 2002$.

[16] E. Keogh et al. Dimensionality reduction for fast similarity search in large time series databases. KAIS, 3(3), 2000

[17] M. Kontaki, A. N. Papadopoulos. Efficient similarity search in streaming time sequences. SSDBM, 2004.

[18] F. Korn et al. Efficiently supporting ad hoc queries in large datasets of time sequences. SIGMOD, 1997.

[19] Z. Liu et al. Locating motifs in time-series data. $P A K D D, 2005$.

[20] A. Natsev et al. Walrus: a similarity retrieval algorithm for image databases. SIGMOD, 1999.

[21] L. M. Ni et al. LANDMARC: Indoor Location Sensing Using Active RFID. ACM Wireless Networks, 2004.

[22] S. Papadimitriou et al. Streaming pattern discovery in multiple time-series. VLDB, 2005.

[23] I. Popivanov and R. J. Miller. Similarity search over time series data using wavelets. ICDE, 2001.

[24] M. H. Protter, C. B. Jr. Morrey. A First Course in Real Analysis. Springer-Verlag, 1977.

[25] N. D. Sidiropoulos, R. Bro. Mathematical programming algorithms for regression-based non-linear filtering. Signal Processing, 1999.

[26] E. J. Stollnitz et al. Wavelets for computer graphics: theory and applications. Morgan Kaufmann Publishers Inc., 1996.

[27] M. Vlachos et al. Discovering similar multidimensional trajectories. ICDE, 2002.

[28] R. Weber et al. A quantitative analysis and performance study for similarity-search methods in high-dimensional spaces. VLDB, 1998.

[29] $\mathrm{H}$. Wu et al. Online event driven subsequence matching over financial data streams. SIGMOD, 2004.

[30] W. Xue et al. Contour map matching for event detection in sensor networks, SIGMOD, 2006.

[31] B-K Yi, C. Faloutsos. Fast time sequence indexing for arbitrary $\mathrm{L}_{p}$ norms. $V L D B, 2000$.

[32] B.-K. Yi et al, and A. Biliris. Online data mining for coevolving time sequences. $I C D E, 2000$.

[33] Y. Zhu, D. Shasha. Efficient elastic burst detection in data streams. SIGKDD, 2003.

[34] Y. Zhu, D. Shasha. Warping indexes with envelope transforms for query by humming. SIGMOD, 2003. 